\title{
Comparison of Cerebrospinal Fluid Biomarkers for Differential Diagnosis of Acute Bacterial and Viral Meningitis with Atypical Cerebrospinal Fluid Characteristics
}

\author{
Sérgio Monteiro de Almeida Suélen Maria Parizotto Furlan \\ Arianne Maris Munhoz Cretella Bruna Lapinski Keite Nogueira \\ Laura Lucia Cogo Luine Rosele Renaud Vidal Meri Bordignon Nogueira \\ Hospital de Clínicas, Universidade Federal do Paraná, Curitiba, Brazil
}

\section{Significance of the Study}

- The ability of cerebrospinal fluid (CSF) biomarkers to discriminate between acute bacterial and viral meningitis was studied.

- This study included microbiologically confirmed acute meningitis and atypical CSF characteristics.

- Operational characteristics of CSF lactate were better than those of other biomarkers.

\section{Keywords}

Acute meningitis · Bacterial meningitis · Cerebrospinal fluid · Lactic acid $\cdot$ Lactate $\cdot$ Viral meningitis

\footnotetext{
Abstract

Objective: Several cerebrospinal fluid (CSF) biomarkers are used to distinguish between acute bacterial meningitis (BM) and viral meningitis (VM). We compared the ability of lactate and glucose (GL) in CSF and the CSF/blood GL ratio to distinguish between acute BM and VM with typical and atypical CSF characteristics. Methods: Three hundred and twentyfour CSF reports were included, which were distributed as the acute BM, VM, and normal control groups ( $n=63,139$, and 122, respectively). Results: Lactate level in the CSF of acute BM group was 4-fold higher than that in the acute VM
}

and control groups $(p<0.0001)$. CSF lactate presented higher specificity (92\%) and negative predictive value (94\%) compared to CSF GL and CSF/blood GL ratio in distinguishing acute BM and VM. Definitive acute BM or VM with atypical CSF cell characteristics was observed in 23.2 and $21.6 \%$ of samples, respectively, and these groups showed reduced performance of characteristics of all CSF biomarkers. CSF lactate showed better operational characteristics than those of CSF GL and CSF/blood GL ratio, presenting the highest positive likelihood ratio, and thus aided in the differential diagnosis of VM with atypical CSF. Conclusion: The CSF lactate assay can be routinely used in laboratories as a rapid, automated, and easy method that is independent of lactate blood levels. (c) 2019 The Author(s)

Published by S. Karger AG, Basel

\begin{tabular}{ll}
\hline KARGER & $\begin{array}{l}\text { Ko 2019 The Author(s) } \\
\text { Published by S. Karger AG, Basel Operger }\end{array}$ \\
karger@karger.com & This is an Open Access article licensed under the Creative Commons \\
www.karger.com/mpp & $\begin{array}{l}\text { Attribution-NonCommercial-4.0 International License (CC BY-NC) } \\
\text { (http://www.karger.com/Services/OpenAccessLicense), applicable to } \\
\text { the online version of the article only. Usage and distribution for com- } \\
\text { mercial purposes requires written permission. }\end{array}$
\end{tabular}

Sérgio Monteiro de Almeida

Hospital de Clínicas, UFPR, Seção de Virologia

Setor Análises Clínicas Rua Padre Camargo

280 Curitiba PR 80060-240 (Brazil)

E-Mail sergio.ma@ufpr.br 


\section{Introduction}

The differential diagnosis between acute bacterial meningitis (BM) and viral meningitis (VM) is crucial for treatment and prognosis, although it is difficult in some cases because the clinical characteristics and classical cerebrospinal fluid (CSF) biochemistry and cell characteristics may overlap. In addition, identification of the etiological agent is not always possible [1].

Several CSF biomarkers can be used to differentiate acute BM and VM $[1,2]$. CSF lactate is considered a good biomarker for differentiating BM from VM at a cutoff of $3.5 \mathrm{mmol} / \mathrm{L}[1]$ and has the advantage of being independent of blood lactate level.

In this study, we aimed to compare the ability of CSF biomarkers (lactate, GL, CSF/blood GL ratio, and predominance of neutrophils) to discriminate between acute BM and VM with typical and atypical CSF cell and biochemistry characteristics. To the best of our knowledge, no study on atypical CSF characteristics has been reported before. Furthermore, we attempted to determine the effect of red blood cells (RBCs) in the CSF on the lactate concentration in the CSF after traumatic lumbar puncture (LP).

\section{Subjects and Methods}

\section{Subjects}

This retrospective study was conducted on CSF reports from the Clinical Pathology Laboratory of the Complex Hospital de Clinicas, Universidade Federal do Paraná. The study was approved by the Institutional Research Review Board of the Complex Hospital de Clınicas, Universidade Federal do Paraná, Brazil.

All CSF samples were collected for clinical purposes. Patients with clinical suspicion of acute BM or VM were selected randomly based on microbiological identification of the etiological agent (Fig. 1). The inclusion criteria were as follows: available CSF samples from patients with clinical suspicion of acute meningitis, with identified etiological agent (bacteria or virus) and samples in which CSF lactate had been quantified. In cases with $>1$ CSF sample, only the first was included.

\section{Sample Collection}

In the BM group, 35 (56\%) and 28 (44\%) samples were collected by LP and ventricular puncture, respectively; the corresponding values in the VM group were 134 (96.4\%) and 3 (2.15\%), respectively, and $2(1.45 \%)$ by suboccipital (cisternal) puncture. In the control group, $122(100 \%)$ samples were obtained by LP.

During the period of this study (1996-2015), 435 CSF samples were analyzed; 324 samples fulfilled the inclusion criteria (Fig. 1). The samples were distributed to the following groups; CSF samples without differential white blood cell (WBC) count were excluded from the analysis:
Group 1, definitive acute BM $(n=63)$ : all cases were caused by bacteria identified by culture. Thirty-four cases (54\%) were due to gram-positive bacteria: Streptococcus pneumoniae $(n=18)$, coagulase-negative staphylococci $(n=9)$, Staphylococcus aureus $(n=4)$, Group D Streptococcus $(n=1)$, Streptococcus viridans $(n=1)$, and Streptococcus sanguinis $(n=1) ; 29$ cases $(46 \%)$ were gram-negative bacteria: Neisseria meningitidis $(n=9)$, Enterobacter cloacae $(n=$ $6)$, Klebsiella pneumoniae $(n=5)$, Acinetobacter $(n=2)$, Escherichia coli $(n=2)$, Haemophilus influenzae $(n=1)$, Enterobacter aerogenes $(n=1)$, Klebsiella oxytoca $(n=1)$, Proteus mirabilis $(n=2)$, and Pseudomonas stutzeri $(n=1)$. To study the accuracy of the CSF biomarkers in diagnosing atypical acute BM, this group was further subdivided into (a) definitive acute BM with typical CSF cell characteristics, that is, increase in total WBC with predominance of neutrophils ( $n=43 ; 76.8 \%$ ), and (b) definitive acute BM with atypical CSF cell characteristics, that is, increase in total WBC with predominance of lymphocytes or normal CSF WBC count $(\leq 5 \times$ $\left.10^{6} / \mathrm{L}, n=13,23.2 \%\right)$.

Group 2, definite acute VM or encephalitis $(n=139)$ : viruses were identified using polymerase chain reaction. Seventy-eight cases $(56.1 \%)$ of herpesvirus family were identified: cytomegalovirus $(n=9)$, Epstein-Barr virus $(n=22)$, human herpes virus-6 (HHV-6, $n=10$ ), herpes simplex virus (HSV, $n=28$ ), and varicella-zoster virus $(n=9)$; and enterovirus was identified in 61 cases (43.9\%). To study the accuracy of the CSF biomarkers in detecting atypical acute viral central nervous system infection, this group was further divided into (a) definite acute VM or encephalitis with typical CSF cell characteristics, that is, increase in total WBC with predominance of lymphocytes $(n=76,78.4 \%)$, and (b) definite acute VM or encephalitis with atypical CSF characteristics, that is, increase in total WBC with predominance of neutrophils $(n=21$, $21.6 \%$ ).

Group 3, CSF normal control group $(n=122)$ : CSF samples with clinical suspicion of acute meningitis not proved by CSF examination and clinical evolution, with normal CSF cellular and biochemical features (total protein and GL) such as WBC $\leq 5 \times$ $10^{6} / \mathrm{L}, \mathrm{GL} \geq 2.66 \mathrm{mmol} / \mathrm{L}$, and total protein $\leq 0.45 \mathrm{~g} / \mathrm{L}$.

\section{CSF Biochemistry and Cytology}

Lactate was quantified in CSF and plasma using amperometry (RAPID Point 500, Siemens, NY, USA). CSF total protein was quantified using benzethonium chloride, and GL levels in the CSF and plasma were determined using hexokinase/G-6-PDH (both from Architect, Abbott, IL, USA). The total CSF WBC $\times 10^{6} / \mathrm{L}$ was quantified in fresh uncentrifuged CSF in a Fuchs-Rosenthal chamber. CSF pleocytosis was defined as a WBC count $>5$ cells $/ \times 10^{6} / \mathrm{L}$. Differential WBC counts were performed by standard laboratory methods. CSF xanthochromia was assessed by visual inspection and quantified using the color index $[3,4]$.

\section{Identification of Etiological Agents}

All CSF samples were subjected to direct microscopic examinations (Gram stain smear) and culturing. CSF specimens were inoculated into agar plates ( $5 \%$ sheep blood agar and supplemented chocolate agar plates) and incubated at $37^{\circ} \mathrm{C}$ for $24-48 \mathrm{~h}$. For positive cultures, the bacteria were identified using a VITEK ${ }^{\circledR} 2$ compact system (BioMérieux Vitek, Inc., Hazelwood, MO, USA). Viruses were identified using polymerase chain reaction for enterovirus, HSV-1, HSV-2, cytomegalovirus, Epstein-Barr virus, varicella-zoster virus, HHV-8, HHV-6A, HHV-6B, and HHV-7 [5]. 
Fig. 1. Standards for reporting of diagnostic accuracy studies diagram showing flow of participants for the validation of CSF lactate $(\mathrm{mmol} / \mathrm{L}), \mathrm{GL}(\mathrm{mg} / \mathrm{dL})$, and $\mathrm{CSF} /$ blood GL ratio (index tests) in CSF samples for the differential diagnosis of acute meningitis, bacterial or viral. Reference standards were CSF culture or polymerase chain reaction, in which the bacteria or virus was identified, respectively. CSF, cerebrospinal fluid; WBC, white blood cell; $\mathrm{BM}$, bacterial meningitis; VM, viral meningitis.

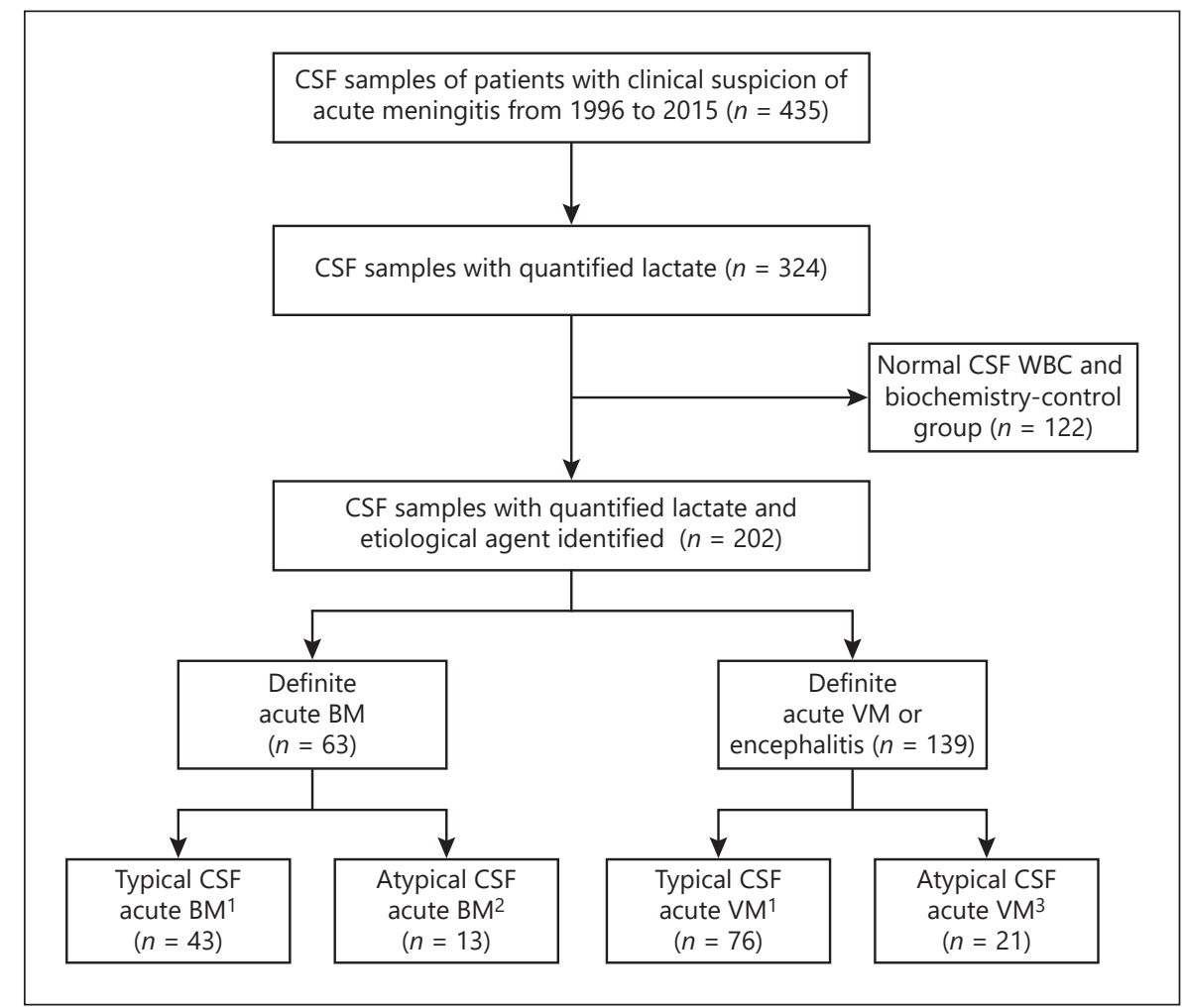

Calculation of Best Discriminating Value of Biomarkers

The receiver operating characteristic curve, constructed by Statistical Package for the Social Sciences version 22 (IBM Corp., Armonk, NY, USA), was used to evaluate the ability of CSF lactate, GL, and CSF/blood GL ratio to accurately classify patients with acute $\mathrm{BM}$ and without disease and establish the best cutoff [6].

\section{Statistical Analyses}

The results were presented as the median (interquartile range), number, and percentage, as appropriate. Categorical variables were compared between groups using Fisher's exact test and continuous variables were compared using Mann-Whitney or Kruskal-Wallis test for nonparametric data, as appropriate. Correlations between variables were calculated using Spearman's rankorder correlation. Results were considered significant at the 5\% alpha level. The concordance between the diagnostic tests was determined using the kappa statistic (K) [7].

\section{Performance Characteristics of CSF for Acute Meningitis}

The performance characteristics of the CSF biomarkers for acute meningitis were calculated using positive CSF culture for bacteria as the reference method. The following performance characteristics were calculated: sensitivity, specificity, accuracy (efficiency), positive predictive value, negative predictive value, Youden's index $(J)=([$ sensitivity + specificity $]-1)$, error ratio $=$ (false positive $[\mathrm{FP}]+$ false negative $[\mathrm{FN}]$ )/true positive (TP) [14], clinical utility index positive (CUI+), and CUI negative (CUI-, screening accuracy) $[8,9]$. CUI was classified as follows: utility excellent, $\geq 0.81$; good, $\geq 0.64$; fair, $\geq 0.49$; poor, $\leq 0.49$; and very poor, $\leq 0.36[8,9]$. The positive and negative likelihood ratios (LR+ and LR-, respectively) were calculated $[6,10,11]$. The posttest probability for a positive and negative test was calculated using the Fagan's nomogram $[12,13]$, considering the pretest probability of acute $\mathrm{BM}$ to be $45 \%$ [14]. FP rate $(\%)=100$ - positive predictive value; presumptive positive $=(\mathrm{TP}+\mathrm{FP}) /$ total; detection rate $=\mathrm{TP} /$ total; error ratio $=(\mathrm{FP}+\mathrm{FN}) / \mathrm{TP}$; and combined error $=(\mathrm{FP}+\mathrm{FN}) /$ total [15].

\section{Results}

Demographic features, CSF biochemistry, and cell characteristics of all study groups are shown in Tables 1 and 2 and Figure 2. The groups were comparable in age and sex.

\section{CSF Lactate in Groups with CSF Atypical CSF \\ Characteristics}

Thirteen cases (23.2\%) were classified as definitive acute BM with atypical CSF cell characteristics $(\leq 5$ cells $\left./ \times 10^{6} / \mathrm{L}\right) ; 5$ samples in this group had normal CSF WBC count, which represented $8.9 \%$ of all cases of BM. These patients might have received or were on antibiotic therapy, although this information was not available. 
Table 1. Epidemiological, cytological, and biochemical characteristics in CSF samples of three groups studied

\begin{tabular}{|c|c|c|c|c|}
\hline & Bacterial & Viral & Control & $p$ value \\
\hline Number & 63 & 139 & 122 & - \\
\hline Age, years & $25(6-48.5)$ & $20.5(11.5-47.5)$ & $22.5(4.5-49.50)$ & 0.19 \\
\hline \multicolumn{5}{|l|}{ CSF } \\
\hline Color index & $0.3(0-1.0)$ & 0 & 0 & $<0.0001$ \\
\hline $\mathrm{RBC} \times 10^{6} / \mathrm{L}$ & $175(20-1,935)$ & $8.1(2.0-51.5)$ & $1.2(0.3-10.8)$ & $<0.0001$ \\
\hline $\mathrm{WBC}>5 \times 10^{6} / \mathrm{L}, n(\%)$ & $50 / 56(89)$ & $104 / 139(74.8)$ & $0 / 122$ & $<0.0001$ \\
\hline$\%$ Neutrophils & $83(74-95)$ & $10(2-38.5)$ & - & $<0.0001$ \\
\hline \% Lymphocytes & $15(4-27)$ & $82(56.5-93)$ & - & $<0.0001$ \\
\hline $\mathrm{GL} \mathrm{mmol} / \mathrm{L}$ & $0.86(0.28-3.02)$ & $3.22(2.66-3.8)$ & $3.55(3.22-4.02)$ & $<0.0001$ \\
\hline $\mathrm{GL}<2.66 \mathrm{mmol} / \mathrm{L}, n(\%)$ & $43 / 58(74.1)$ & $33 / 139(23.7)$ & $5 / 122(4.1)$ & $<0.0001$ \\
\hline $\mathrm{TP} \mathrm{g} / \mathrm{L}$ & $1.91(0.67-3.76)$ & $0.48(0.32-0.92)$ & $0.26(0.17-0.34)$ & $<0.0001$ \\
\hline $\mathrm{GL} \mathrm{mmol/L}$ & $6.11(5.16-7.63)$ & $5.72(4.72-6.88)$ & $5.38(4.83-6.72)$ & 0.24 \\
\hline Lactate $\mathrm{mmol} / \mathrm{L}$ & $1.9(1.15-2.8)$ & $1.56(1.12-2.34)$ & $1.4(1.2-1.8)$ & 0.35 \\
\hline \multicolumn{5}{|l|}{ CSF/blood } \\
\hline GL & $0.30(0.04-0.55)$ & $0.56(0.42-0.67)$ & $0.7(0.58-0.78)$ & $<0.0001$ \\
\hline GL CSF/blood <0.6, $n(\%)$ & $31 / 37(83.8)$ & $65 / 104(62.5)$ & $19 / 69(27.5)$ & $<0.0001$ \\
\hline
\end{tabular}

Data presented in median (IQR) or $n(\%)$, as appropriate. (1) Total protein increased in accordance with reference values by local CSF puncture: ventricular $0.05-0.15 \mathrm{~g} / \mathrm{L}$; suboccipital $0.10-0.25 \mathrm{~g} / \mathrm{L}$, and lumbar $0.15-0.45 \mathrm{~g} / \mathrm{L}$.

CSF, cerebrospinal fluid; WBC, white blood cells; RBC, red blood cells; TP, total proteins; GL, glucose; IQR, interquartile range.

Definite acute VM or encephalitis with atypical CSF characteristics was observed in 21 samples $(21.6 \%)$. The viruses identified were enterovirus $(n=14,67 \%)$ and members of the herpesvirus family $(n=7,33 \%)$. CSF lactate levels in typical and atypical acute BM or VM cases are shown in Table 2 and Figure $2 b$.

The CSF lactate level in the atypical acute BM group was comparable with that in atypical and typical VM groups; there was no difference between the groups with VM (all $p>0.05$ ). Atypical CSF samples of acute BM with increased CSF WBC count $(n=8)$ showed median (interquartile range) of CSF lactate level of 3.55 (2.5-6.35). Compared to levels in groups with typical and atypical $\mathrm{VM}$, the CSF lactate level was higher in bacterial atypical meningitis and increase of CSF WBC count $(p<0.05$; Table 2, Fig. 2).

\section{Calculation of Best Cutoff Points for CSF Lactate and GL}

The best cutoff values of CSF lactate and GL for distinguishing between $\mathrm{BM}$ and $\mathrm{VM}$ and the comparison of the area under the curve (AUC) of the receiver operating characteristic curves of the CSF biomarkers studied are shown in Figure 3.

\section{Performance Characteristics of CSF Biomarkers}

The performance characteristics of CSF lactate, GL, CSF/blood GL ratio, CSF neutrophil predominance, and their associations in distinguishing between acute BM and VM (overall) and with atypical CSF are shown in Tables 3 and 4 , respectively.

The posterior probabilities for a positive and negative test result for lactate, GL, WBCs in CSF, and their associations in the groups with definite acute meningitis and atypical acute BM to discriminate between BM and VM are shown in Figure 4.

\section{Concordance between CSF Biomarkers}

There was moderate concordance between CSF lactate and GL levels and CSF lactate and predominance of neutrophils in the CSF (Kappa index \pm SD; 95\% CI was 0.537 \pm 0.058 [0.423-0.651] and $0.503 \pm 0.074$ [0.358-0.648] respectively). In contrast, there was poor concordance between CSF lactate and CSF/blood GL ratio (0.175 \pm 
Table 2. Epidemiological, cytological, and biochemical characteristics of CSF of the groups with typical and atypical acute meningitis

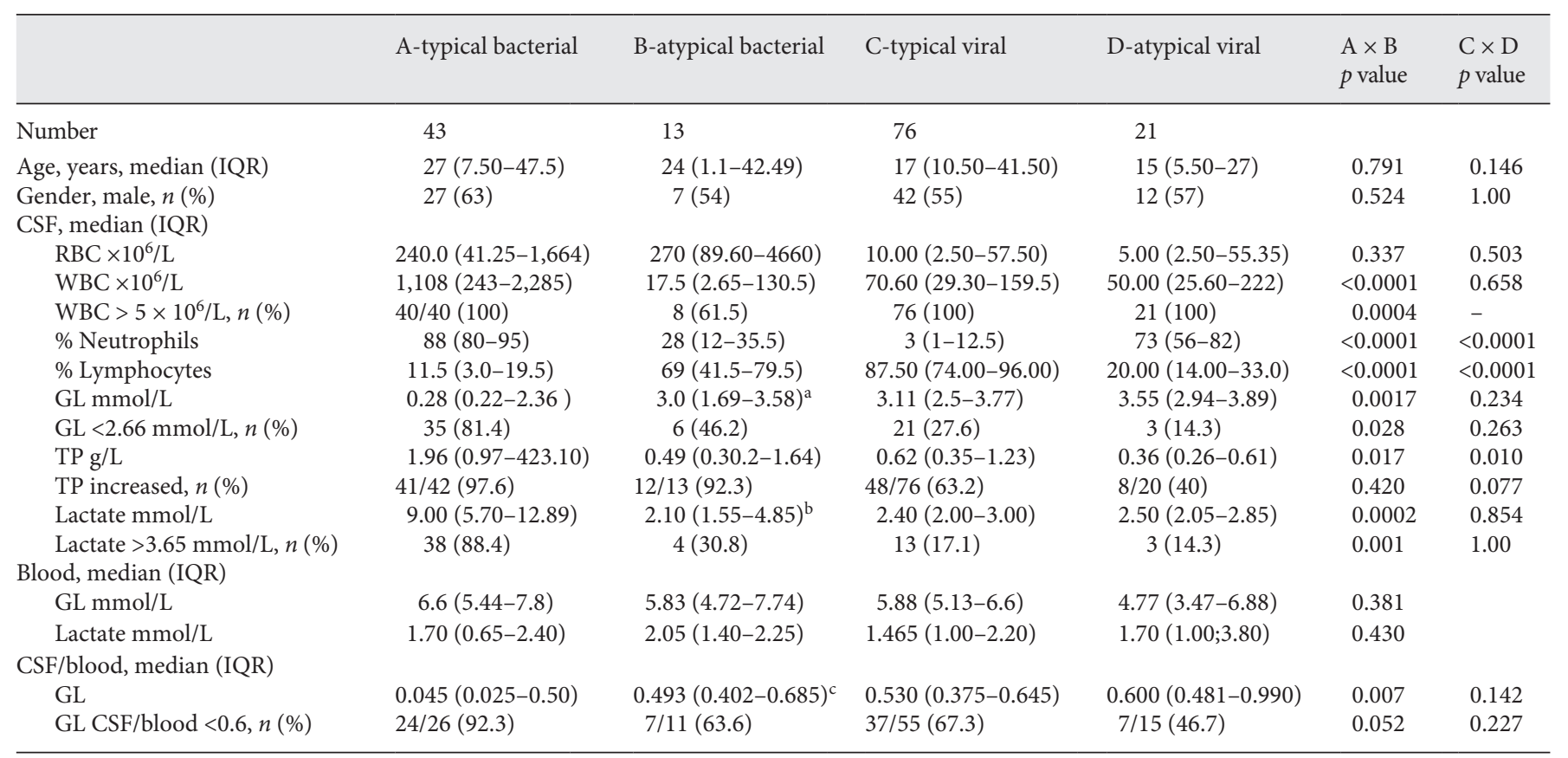

${ }^{a}$ Only atypical CSF samples of acute BM showing increase in CSF WBC number were considered $(n=8)$. CSF GL was 2.75 (1.5-3.27) mmol/L compared to the groups with typical and atypical VM ( $p=0.326$ and 0.1073 , respectively). Compared to the group with typical BM, $p=0.017$.

${ }^{\mathrm{b}}$ Only atypical CSF samples of acute BM showing increase in CSF WBC number were considered $(n=8)$. CSF lactate was 3.55 (2.5-6.35) mmol/L compared to the groups with typical and atypical VM $(p=0.026$ and 0.043 , respectively). Compared to the group with typical $\mathrm{BM}, p=0.027$.

${ }^{c}$ Only atypical CSF samples of acute BM with increase in CSF WBC were considered. CSF and CSF/blood GL ratio was 0.46 (0.26-0.67) compared to groups with typical and atypical VM ( $p=0.297$ and 0.067 , respectively). Compared to the group with typical BM, $p=0.041$.

CSF, cerebrospinal fluid; RBC, red blood cells; WBC, white blood cells; GL, glucose; TP, total proteins; BM, bacterial meningitis; VM, viral meningitis; $\mathrm{IQR}$, interquartile range.

$0.060[0.057-0.293])$, GL and predominance of neutrophils in CSF $(0.321 \pm 0.081$ [0.163-0.479]), and CSF/ blood GL ratio and predominance of neutrophils (0.044 \pm 0.094 [-0.141 to 0.229$])$.

\section{Correlations between CSF Lactate and Other \\ Biomarkers}

There was strong positive correlation between CSF lactate and CSF WBC $\left(R_{\mathrm{s}}=0.63\right.$ [95\% CI 0.56-0.69]); weak positive correlation between CSF lactate and CSF $\mathrm{RBC}$ or color index $\left(R_{\mathrm{s}}=0.30[0.20-0.40]\right.$ and $R_{\mathrm{s}}=0.35$ [0.24-0.45], respectively); and weak negative correlation between CSF lactate and CSF GL or the CSF/blood GL ratio $\left(R_{\mathrm{s}}=-0.29[-0.39\right.$ to -0.18$]$ and $R_{\mathrm{s}}=-0.34$ [ -0.46 to $-0.21]$, respectively), all $p<0.0001$. There was no correlation between CSF and blood lactate levels $(p=0.069)$.

\section{CSF Lactate Levels by Type of Bacteria or Virus}

There was no difference in CSF lactate levels between the groups with gram-positive or gram-negative bacteria in the CSF. In the group with acute BM caused by grampositive bacteria, CSF lactate was 7.45 (3.20-14.60) $\mathrm{mmol} / \mathrm{L}$, whereas it was $7.00(3.80-11.95) \mathrm{mmol} / \mathrm{L}$ in the gram-negative bacteria group $(p=0.736)$. In the group with acute BM caused by gram-positive bacteria, 25 samples $(73.5 \%)$ showed CSF lactate $>3.65 \mathrm{mmol} / \mathrm{L}$, and in the gram-negative bacteria group, there were 23 (79.3\%, $p=0.768$ ). There was no difference in CSF lactate levels between the groups with VM caused by enterovirus and herpesvirus family members; CSF lactate was 2.2 (1.75$2.65) \mathrm{mmol} / \mathrm{L}$ and $2.4(1.8-3.3) \mathrm{mmol} / \mathrm{L}$, respectively $(p=$ $0.060)$.

\section{Discussion}

In the present study, we observed that CSF lactate showed higher operational characteristics than CSF GL did for differentiating between acute BM and VM. The efficiency of GL, lactate, and their association were high- 


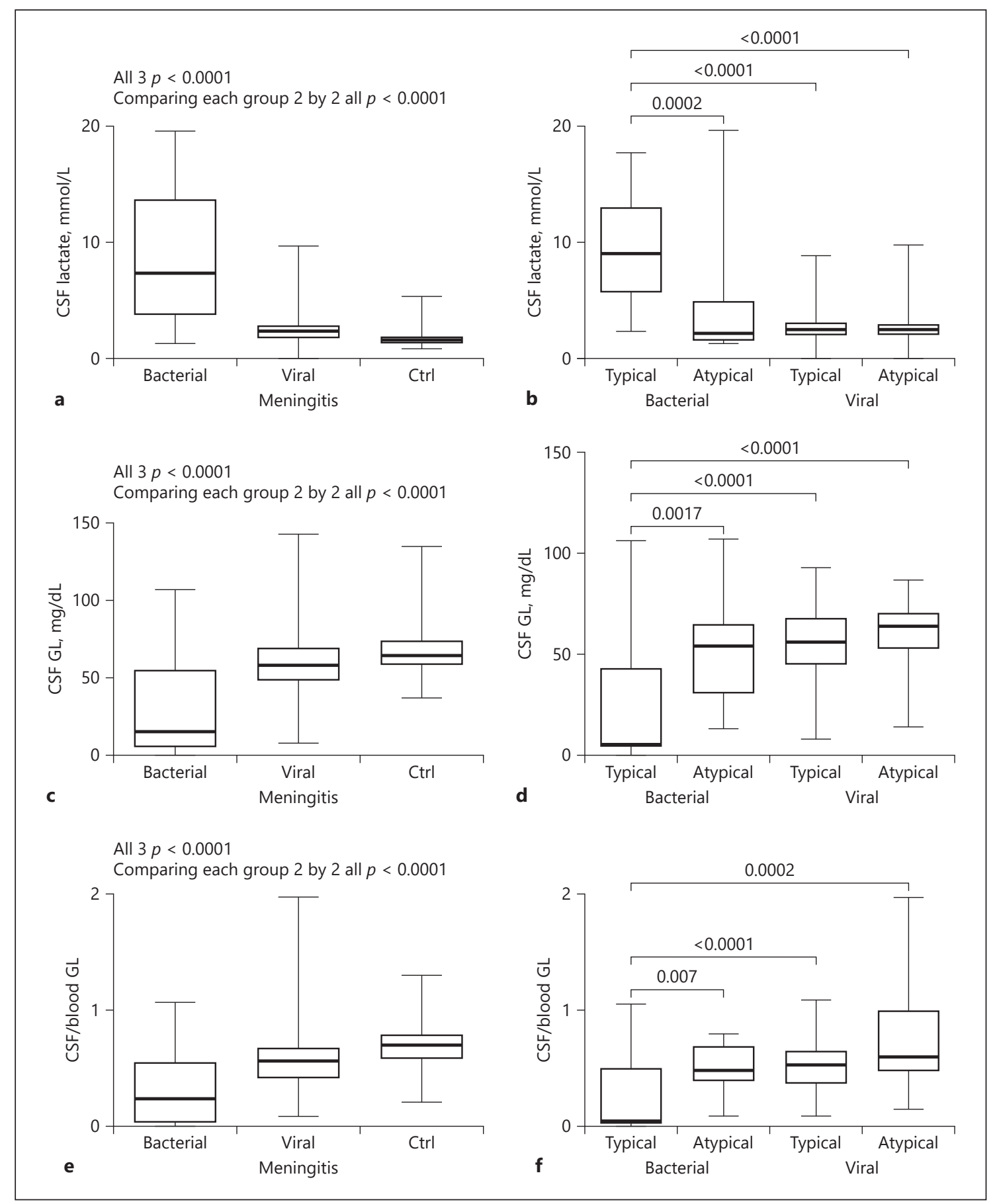

Fig. 2. Levels of CSF biomarkers, lactate $(\mathrm{mmol} / \mathrm{L})$, GL $(\mathrm{mg} / \mathrm{dL})$, and CSF/blood GL ratio in acute BM and VM with typical and atypical CSF characteristics and in the normal control group. Comparisons between acute meningitis and the normal control group (Ctrl): CSF lactate (a); GL (c); CSF/blood GL ratio (e); $p<$ 0.0001 between the 3 groups for each biomarker studied; $p<0.0001$ when each group was compared pairwise. Comparisons between acute BM and VM with typical and atypical CSF characteristics: CSF lactate (b); GL (d); CSF/blood GL ratio (f); for the comparisons not indicated in the figure, $p>0.05$. CSF, cerebrospinal fluid; $\mathrm{BM}$, bacterial meningitis; VM, viral meningitis. 


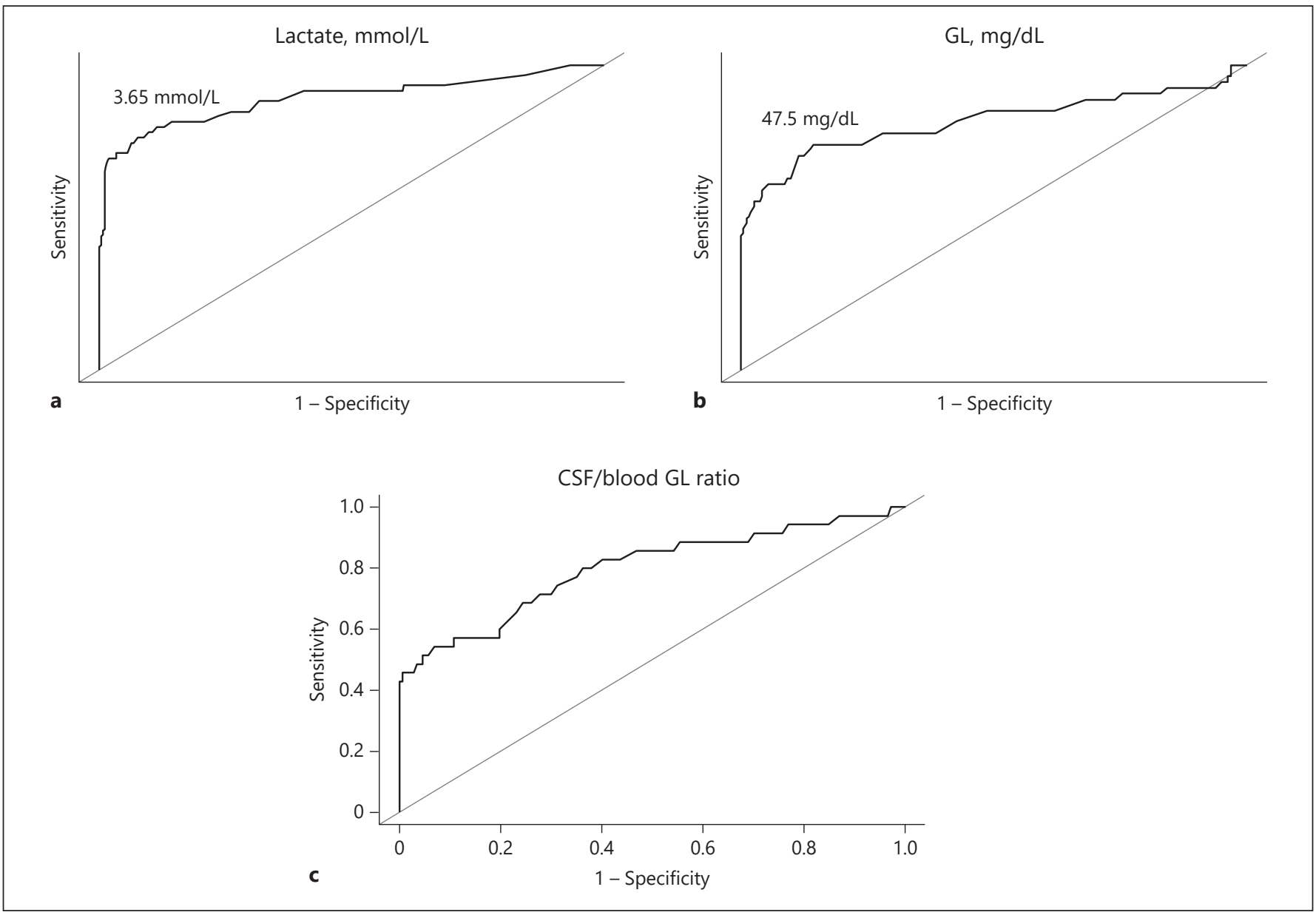

Fig. 3. ROC curve showing best cutoff points for lactate and GL in CSF samples with acute meningitis and etiological agents (bacteria or viral). a ROC curve of CSF lactate indicated best cutoff point of $3.65 \mathrm{mmol} / \mathrm{L}$; values exceeding this are suggestive of acute BM; the
AUC was 0.89 . b ROC curve of CSF GL indicated best cutoff point of $47.5 \mathrm{mg} / \mathrm{dL}(2.64 \mathrm{mmol} / \mathrm{L})$; values lower than this were suggestive of acute BM; the AUC was 0.82. c ROC curve of CSF/blood GL ratio; the AUC was 0.80. GL, glucose; CSF, cerebrospinal fluid. er than the CSF/blood GL ratio and predominance of neutrophils. CSF/blood GL ratio showed high sensitivity, although the specificity was low, similar to the results of a previous study [16]. A systematic review of the assessment of CSF lactate concentration for distinguishing BM from aseptic meningitis reported the mean sensitivity and specificity as 96 and $94 \%$, respectively [17].

The AUC of CSF lactate was 0.89 , which was slightly higher than that of CSF GL (0.82), indicating good overall accuracy [18] for both CSF biomarkers. This was lower than that described previously, where the observed AUCs of CSF lactate ranged from 0.977 to $0.988[17,18]$.

The LR+ and LR- of lactate were 10.00 and 0.26 , respectively. In a systematic review, Sakushima et al. [19] reported that CSF lactate had an LR+ of 22.9 and LR- of
0.07 . They concluded that the low LR-indicated that the lack of CSF lactate is specifically good for discarding a suspicion of BM [19]; another study described similar results [18].

The CSF lactate $\mathrm{LR}+$ was 9.9\%; an $\mathrm{LR}+\geq 10.0$ indicates that a positive test almost confirms the disease. For the other CSF biomarkers, LR+ values were lower than 5.0. $\mathrm{LR}+$ values of approximately 6.0 indicated that the disease was present, and a value of approximately 1.0 indicated that the test could not conclusively confirm the presence of the disease. LR+ $\leq 0.1$ indicated that the disease was practically absent $[6,10,11]$. In a systemic review, the mean LR+ and LR- were calculated to be 14.53 (95\% CI 8.07-26.19) and 0.07 (95\% CI 0.05-0.09), respectively [17]. CSF lactate was lower in the group with atyp- 
Table 3. Performance characteristics of CSF lactate, GL, CSF/blood GL ratio, and association of lactate and GL for differentiating acute BM and VM

\begin{tabular}{|c|c|c|c|c|c|c|c|}
\hline & $\begin{array}{l}\text { Glucose } \\
<2.66 \\
\mathrm{mmol} / \mathrm{L}\end{array}$ & $\begin{array}{l}\text { Lactate } \\
>3.65 \\
\mathrm{mmol} / \mathrm{L}\end{array}$ & $\begin{array}{l}\mathrm{CSF} / \\
\text { blood } \\
\mathrm{GL} \\
<0.6\end{array}$ & Neutrophils* & $\begin{array}{l}\text { GL } \\
\text { and/or } \\
\text { lactate }\end{array}$ & $\begin{array}{l}\text { Lactate } \\
\text { and/or } \\
\text { GL CSF/ } \\
\text { blood }\end{array}$ & $\begin{array}{l}\text { Lactate } \\
\text { and/or } \\
\text { neutrophils* }\end{array}$ \\
\hline $\mathrm{TP}$ & 43 & 48 & 31 & 44 & 53 & 56 & 53 \\
\hline Sensitivity, \% & 74.14 & 76.19 & 83.78 & 84.62 & 84.13 & 88.89 & 84.13 \\
\hline Specificity, \% & 85.44 & 92.34 & 51.45 & 76.92 & 81.61 & 64.37 & 73.38 \\
\hline PPV, \% & 53.09 & 70.59 & 26.96 & 67.69 & 52.48 & 37.58 & 58.89 \\
\hline NPV, $\%$ & 93.70 & 94.14 & 93.68 & 89.74 & 95.52 & 96.00 & 91.07 \\
\hline LRP & 5.090 & 9.94 & 1.73 & 3.67 & 4.57 & 2.49 & 3.16 \\
\hline LRN & 0.30 & 0.26 & 0.32 & 0.20 & 0.19 & 0.17 & 0.22 \\
\hline $\mathrm{CUI+}$ & 0.39 & 0.54 & 0.23 & 0.57 & 0.44 & 0.33 & 0.50 \\
\hline CUI- & 0.80 & 0.87 & 0.48 & 0.69 & 0.78 & 0.62 & 0.67 \\
\hline Youden index & 0.60 & 0.69 & 0.35 & 0.62 & 0.66 & 0.53 & 0.58 \\
\hline False-positive rate, $\%$ & 46.91 & 29.41 & 73.00 & 32.31 & 47.52 & 62.42 & 41.11 \\
\hline Presumptive positive, $\%$ & 0.25 & 0.21 & 0.55 & 0.45 & 0.31 & 0.46 & 0.45 \\
\hline Detection rate, $\%$ & 13.48 & 14.81 & 14.76 & 30.75 & 16.38 & 17.28 & 26.24 \\
\hline Error rate, $\%$ & 123 & 72.92 & 290 & 65.90 & 109 & 179 & 88.70 \\
\hline Combined error, \% & 16.61 & 10.80 & 42.86 & 20.28 & 17.90 & 30.86 & 23.27 \\
\hline Efficiency, \% & 83.39 & 89.20 & 57.14 & 79.72 & 82.10 & 69.14 & 76.73 \\
\hline
\end{tabular}

* Predominance of neutrophils on the groups with BM and VM.

CSF, cerebrospinal fluid; GL, glucose; BM, bacterial meningitis; VM, viral meningitis; PPV, positive predictive value; NPV, negative predictive value; CUI+, clinical utility index positive; CUI-, clinical utility index negative.

Table 4. Performance characteristics of CSF lactate, GL, CSF/blood GL ratio, and association of lactate and GL for differentiating acute BM with atypical CSF and VM

\begin{tabular}{|c|c|c|c|c|c|c|c|}
\hline & $\begin{array}{l}\mathrm{GL} \\
<2.66 \\
\mathrm{mmol} / \mathrm{L}\end{array}$ & $\begin{array}{l}\text { Lactate } \\
>3.65 \\
\mathrm{mmol} / \mathrm{L}\end{array}$ & $\begin{array}{l}\mathrm{CSF} / \\
\text { blood } \\
\mathrm{GL} \\
<0.6\end{array}$ & Neutrophils* & $\begin{array}{l}\text { GL } \\
\text { and/or } \\
\text { lactate }\end{array}$ & $\begin{array}{l}\text { Lactate } \\
\text { and/or } \\
\text { GL CSF/ } \\
\text { blood }\end{array}$ & $\begin{array}{l}\text { Lactate } \\
\text { and/or } \\
\text { neutrophils* }\end{array}$ \\
\hline $\mathrm{TP}$ & 6 & 4 & 8 & 2 & 7 & 8 & 4 \\
\hline Sensitivity, \% & 46.15 & 30.77 & 72.73 & 25.00 & 53.85 & 61.54 & 30.77 \\
\hline Specificity, \% & 85.44 & 92.34 & 51.45 & 76.92 & 81.61 & 64.37 & 73.38 \\
\hline $\mathrm{PPV}, \%$ & 13.64 & 16.67 & 8.70 & 8.70 & 12.73 & 7.92 & 9.76 \\
\hline NPV, \% & 96.96 & 96.40 & 96.74 & 92.11 & 97.26 & 97.11 & 91.89 \\
\hline LRP & 3.17 & 4.02 & 1.50 & 1.08 & 2.93 & 1.73 & 1.16 \\
\hline LRN & 0.63 & 0.75 & 0.53 & 0.97 & 0.57 & 0.60 & 0.94 \\
\hline CUI+ & 0.06 & 0.05 & 0.06 & 0.02 & 0.07 & 0.05 & 0.03 \\
\hline CUI- & 0.83 & 0.89 & 0.50 & 0.71 & 0.79 & 0.63 & 0.67 \\
\hline Youden index & 0.32 & 0.23 & 0.24 & 0.02 & 0.35 & 0.26 & 0.04 \\
\hline False-positive rate, $\%$ & 86.36 & 83.33 & 91.30 & 91.30 & 87.27 & 92.08 & 90.24 \\
\hline Presumptive positive, $\%$ & 16.06 & 8.76 & 50.00 & 23.23 & 20.07 & 10.22 & 26.97 \\
\hline Detection rate, $\%$ & 2.19 & 1.46 & 4.35 & 2.02 & 2.55 & 2.92 & 2.63 \\
\hline Error rate, $\%$ & 7.50 & 7.25 & 10.90 & 13.50 & 6.29 & 12.25 & 9.0 \\
\hline Combined error, $\%$ & 16.42 & 10.58 & 47.28 & 27.27 & 19.71 & 35.77 & 23.68 \\
\hline Efficiency, \% & 83.58 & 89.42 & 52.72 & 72.73 & 80.29 & 64.23 & 69.74 \\
\hline
\end{tabular}

* Predominance of neutrophils on the groups with BM and VM.

CSF, cerebrospinal fluid; GL, glucose; VM, viral meningitis; PPV, positive predictive value; NPV, negative predictive value; $\mathrm{CUI}+$, clinical utility index positive; $\mathrm{CUI}-$, clinical utility index negative; $\mathrm{BM}$, bacterial meningitis. 


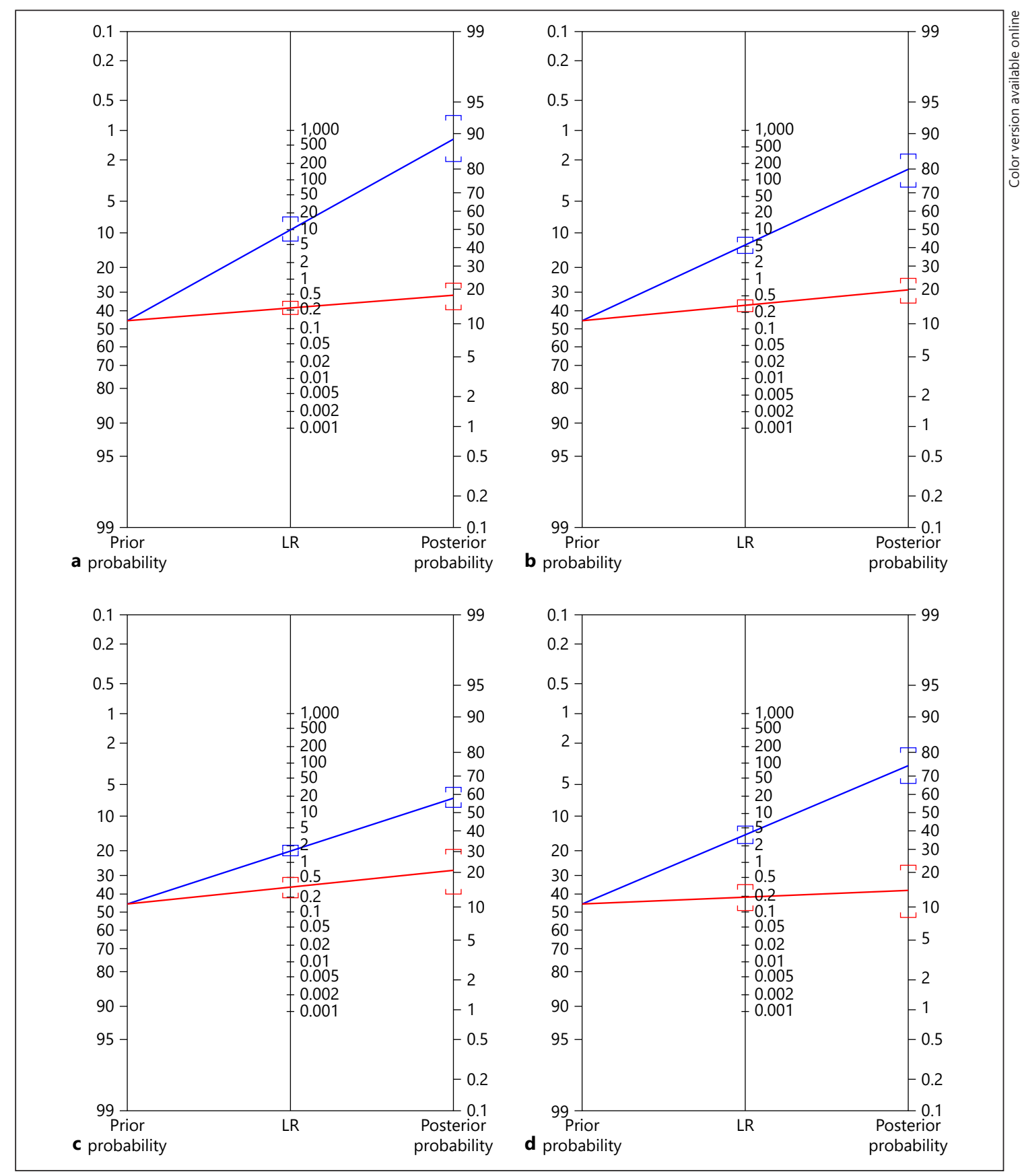

Fig. 4. Fagan's nomogram was used to calculate posterior probability for lactate, GL, WBC in CSF, and their associations for positive and negative tests in the groups with definite acute meningitis and with atypical acute BM. Prior probability (odds) of acute BM: $45 \%$. Posterior odd $=$ Prior odd $\times$ LR. For a positive test (blue line): $(\mathbf{a})$ CSF lactate (>3.65 mol/L). LR+ was 9.90 (95\% CI 5.90-17); posterior probability (odds) was $89 \%$ (95\% CI 83-93\%). Approximately 1 in 1.1 with positive test had acute BM. b CSF GL $(<48 \mathrm{mg} / \mathrm{dL})$. LR+: 5.08 (95\% CI 3.50-7.35); posterior probability (odds): $81 \%$ (95\% CI 74-86\%). Approximately 1 in 1.2 with positive test had acute BM. c CSF/blood GL ratio (<0.6). LR+: 1.72 (95\% CI 1.402.12 ); posterior probability (odds) was $58 \%$ (95\% CI 53-63\%). Ap- proximately 1 in 1.7 with positive test had acute BM. d Predominance of neutrophils in CSF. LR+: 3.66 (95\% CI 2.41-5.55); posterior probability (odds): $75 \%$ (95\% CI 66-82\%). Approximately 1 in 1.3 with positive test had acute BM. Analyzing only the group with atypical BM CSF (graphic not showed): CSF lactate (>3.65 $\mathrm{mol} / \mathrm{L}$ ), posterior probability (odds) was 77\% (95\% CI 64-86\%). CSF GL $(<2.66 \mathrm{~mol} / \mathrm{L})$, posterior probability (odds) was $72 \%(95 \%$ CI $63-80 \%)$. CSF/blood GL ratio $(<0.6)$, posterior probability (odds) was 55\% (95\% CI 49-61\%). Predominance of neutrophils in CSF, posterior probability (odds) was 47\% (95\% CI 31-64\%). The red line indicates the posterior odd for a negative test. LR, likelihood ratio. 
ical acute BM than it was in the group with typical acute $\mathrm{BM}$ and was comparable to that in typical or atypical VM, showing its limited value in differentiating atypical acute BM from typical VM. However, it could still be used to differentiate typical acute BM and atypical VM. The same conclusions are applicable to CSF GL and CSF/blood GL. CSF lactate was higher in the group with atypical acute $\mathrm{BM}$ with increased CSF WBC number than it was in the groups with atypical VM.

The operational characteristics were low for all biomarkers and associations studied in the group with atypical acute BM. Other studies have indicated that in partially treated BM, CSF lactate with a limit $>3.20 \mathrm{mmol} / \mathrm{L}$ classified $88 \%$ of treated BM with a sensitivity and specificity of 83 and $91 \%$, respectively. The sensitivity of the lactate concentration decreased to $72 \%$ in patients who had already been treated with antibiotics, similar to the other biomarkers [16]. CSF lactate was the most reliable variable for differentiating BM after 4 days of antibiotic treatment for lymphocytic meningitis.

Differential diagnosis of BM and VM can be difficult in some cases as data on classical CSF biochemistry and cell characteristics are not always available. A CSF sample with normal appearance, cytology, and biochemical characteristics does not rule out the presence of bacteria, which can occur in $12-30 \%$ of cases of meningitis [20]. Partially treated BM exhibits atypical cell patterns, and the positivity of classic bacteriological diagnostic methods is reduced from 50 to $20 \%$ [21-23]. CSF characteristics of BM can resemble those of VM in immunosuppressed patients [1]. In contrast, VM, especially enterovirus-induced, can exhibit a predominance of neutrophils during the first $6 \mathrm{~h}$ of infection, albeit with normal CSF GL concentration [1]. Approximately $50 \%$ of patients with granulocytopenia who have acute $\mathrm{BM}$ have $<5$ cells/ $\mathrm{mm}^{3}$ in the CSF, which can be confirmed by a positive Gram stain, CSF culture, and serological results [1]. We did not observe any correlation between CSF and blood lactate, corroborating previous observations $[1,24]$. As traumatic LP is common in CSF samples in a routine laboratory, the effect of any resulting CSF RBC on CSF lactate concentrations was assessed. We observed a positive weak correlation between CSF lactate and CSF RBC or color index.

This study is different from previously published studies [16-18] because all the selected samples of acute meningitis were microbiologically confirmed. This could explain the differences between our findings and those previously reported in the literature. Furthermore, we calculated the operational characteristics of definitive acute meningitis with atypical CSF characteristics. The effectiveness of CSF lactate in differentiating BM from VM was compared with that of other conventional CSF biomarkers.

The main limitation of this study was its retrospective design and no longitudinal analysis.

\section{Conclusion}

CSF lactate enabled the differential diagnosis of VM with atypical CSF from BM. It was also useful for the differential diagnosis of BM with increase of CSF WBC and predominance of lymphocytes from VM. However, the results should be interpreted in line with clinical findings and the results of conventional assays including CSF concentrations of protein, cells, and GL, as well as a microbiological analysis of the CSF.

\section{References}

1 McGill F, Heyderman RS, Panagiotou S, Tunkel AR, Solomon T. Acute bacterial meningitis in adults. Lancet. 2016 Dec;388(10063): 3036-47.

2 Nigrovic LE, Kimia AA, Shah SS, Neuman MI. Relationship between cerebrospinal fluid glucose and serum glucose. N Engl J Med. 2012 Feb;366(6):576-8.

3 Arora S, Swadron SP, Dissanayake V. Evaluating the sensitivity of visual xanthochromia in patients with subarachnoid hemorrhage. J Emerg Med. 2010 Jul;39(1):13-6.

4 Edlow JA, Bruner KS, Horowitz GL. Xanthochromia. Arch Pathol Lab Med. 2002 Apr; 126(4):413-5.
5 Vidal LR, Almeida SM, Messias-Reason IJ, Nogueira MB, Debur MC, Pessa LF, et al. Enterovirus and herpesviridae family as etiologic agents of lymphomonocytary meningitis, Southern Brazil. Arq Neuropsiquiatr. 2011 Jun;69(3):475-81.

6 Akobeng AK. Understanding diagnostic tests 3: receiver operating characteristic curves. Acta Paediatr. 2007 May;96(5):644-7.

7 Sim J, Wright CC. The kappa statistic in reliability studies: use, interpretation, and sample size requirements. Phys Ther. 2005 Mar; 85(3):257-68.
8 Mitchell AJ. The clinical significance of subjective memory complaints in the diagnosis of mild cognitive impairment and dementia: a meta-analysis. Int J Geriatr Psychiatry. 2008 Nov;23:1191-1202.

9 Mitchell AJ. Sensitivity $\times$ PPV is a recognized test called the clinical utility index (CUI+). Eur J Epidemiol. 2011 Mar;26(3):251-2.

10 Akobeng AK. Understanding diagnostic tests 2: likelihood ratios, pre- and post-test probabilities and their use in clinical practice. Acta Paediatr. 2007 Apr;96(4):487-91.

11 McGee S. Simplifying likelihood ratios. J Gen Intern Med. 2002 Aug;17(8):646-9. 
12 Sackett DL, Haynes RB. The architecture of diagnostic research. BMJ. 2002 Mar; 324(7336):539-41.

13 Fagan TJ. Letter: nomogram for Bayes theorem. N Engl J Med. 1975 Jul;293(5):257.

14 Hildebrand J, Aoun M. Chronic meningitis: still a diagnostic challenge. J Neurol. 2003 Jun; 250(6):653-60.

15 Galen RS, Gambino SR. Beyond normality: the predictive value and efficiency of medical diagnoses. New York: John Wiley \& Sons 1975. p. 237.

16 Briem H. Comparison between cerebrospinal fluid concentrations of glucose, total protein, chloride, lactate, and total amino acids for differential diagnosis of patients with meningitis. Scand J Infect Dis. 1983;15:277-284.

17 Huy NT, Thao NT, Diep DT, Kikuchi M, Zamora J, Hirayama K. Cerebrospinal fluid lactate concentration to distinguish bacterial from aseptic meningitis: a systemic review and meta-analysis. Crit Care. 2010;14(6): R240.

18 Nazir M, Wani WA, Malik MA, Mir MR, Ashraf Y, Kawoosa K, et al. Cerebrospinal fluid lactate: a differential biomarker for bacterial and viral meningitis in children. J Pediatr (Rio J). 2018 Jan-Feb;94(1):88-92.

19 Sakushima K, Hayashino Y, Kawaguchi T, Jackson JL, Fukuhara S. Diagnostic accuracy of cerebrospinal fluid lactate for differentiating bacterial meningitis from aseptic meningitis: a meta-analysis. J Infect. 2011 Apr;62(4): $255-62$.

20 Talukdar B, Khalil A, Sarkar R, Saini L. Meningococcal meningitis: clinical observations during an epidemic. Indian Pediatr. 1988 Apr; 25:329-334.
21 Feldman WE. Effect of prior antibiotic therapy on concentrations of bacteria in CSF. Am J Dis Child. 1978 Jul;132(7):672-4.

22 Kanegaye JT, Soliemanzadeh P, Bradley JS. Lumbar puncture in pediatric bacterial meningitis: defining the time interval for recovery of cerebrospinal fluid pathogens after parenteral antibiotic pretreatment. Pediatrics. 2001 Nov; 108(5):1169-74.

23 Blazer S, Berant M, Alon U. Bacterial meningitis: effect of antibiotic treatment on cerebrospinal fluid. Am Soc Clin Pathol. 1983 Sep;80: 386-377.

24 de Almeida SM, Faria FL, de Goes Fontes K, Buczenko GM, Berto DB, Raboni SM, et al. Quantitation of cerebrospinal fluid lactic acid in infectious and non-infectious neurological diseases. Clin Chem Lab Med. 2009; 47(6):755-61. 